\title{
Microbiome alterations following solid-organ transplantation: consequences, solutions, and prevention
}

This article was published in the following Dove Press journal: Transplant Research and Risk Management

\author{
Hannah P Rahim ${ }^{1,2, *}$ \\ Michael R Taylor ${ }^{1,2, *}$ \\ Simon A Hirota ${ }^{3}$ \\ Steven C Greenway ${ }^{1,2,4}$ \\ 'Department of Pediatrics, Alberta \\ Children's Hospital Research Institute, \\ Cumming School of Medicine, \\ University of Calgary, ${ }^{2}$ Department of \\ Cardiac Sciences, Libin Cardiovascular \\ Institute of Alberta, Cumming School \\ of Medicine, University of Calgary, \\ ${ }^{3}$ Department of Pharmacology and \\ Physiology, Snyder Institute for \\ Chronic Diseases, Cumming School \\ of Medicine, University of Calgary, \\ ${ }^{4}$ Department of Biochemistry and \\ Molecular Biology, Cumming School \\ of Medicine, University of Calgary, \\ Calgary, AB, Canada
}

*These authors contributed equally to this work

Correspondence: Steven C Greenway Section of Cardiology, Alberta Children's Hospital, 2888 Shaganappi Trail Northwest, Calgary, AB T3B 6A8, Canada

Tel +l 4039555049

Fax + I 403955762 I

Email scgreenw@ucalgary.ca

\begin{abstract}
Improved long-term survival following solid-organ transplantation (SOT) has remained elusive over the past several decades, despite significant advances in early survival. The microbiome refers to the genetic material belonging to microbes that live in an ecological balance with the human host, and its importance in human health is increasingly recognized. Extensive research pertaining to the human microbiome has demonstrated that compositional changes in the microbiome can contribute to such diseases as inflammatory bowel disease, metabolic syndrome, and (recently) many of the comorbidities that develop after SOT. It is suggested that the microbiome may be an important environmental variable that could influence health outcomes after SOT. Many factors related to SOT, including end-stage organ disease, surgery, and the use of antimicrobial prophylaxis and immunosuppressive drugs, have been shown to affect microbial composition and function negatively. These alterations could compromise health outcomes after SOT through the dysregulation of important host-microbe interactions, including the modulation of local and systemic host immune function by the gut microbiome, and could contribute to morbidity and even allograft rejection. Such interventions as synbiotic therapy and fecal microbiota transplantation have the potential to prevent or reverse disruption of the microbiome related to SOT and thereby improve the longevity of transplant recipients. Although microbiome research is still a relatively new field, progress is accelerating exponentially. Future research on host-microbiome interactions in the context of SOT will facilitate the development of microbiome-directed treatments to improve patient outcomes on pre- and post-SOT.
\end{abstract}

Keywords: transplantation, immunosuppression, dysbiosis, immune system, microbiome

\section{Plain language summary}

Since the development of effective immunosuppression, major improvements have occurred in early health outcomes following solid-organ transplantation (SOT) related to medical and surgical advances. However, long-term survival rates after SOT have remained relatively constant. One recently recognized variable that may impact health outcomes after SOT is the microbiome. The microbiome refers to the community of microbes and their genes that inhabit the human body and live in an ecological balance with the host. The microbiome has been shown to play a role in many of the comorbidities that affect patients after SOT, and because the microbiome interacts with the host immune system, it may also play a role in rejection of the transplanted organ. Furthermore, patients before and after SOT are exposed to numerous drugs and procedures that may have a negative impact on the microbiome and alter its composition and function, with potential consequences for the transplant recipient. This review explores the bidirectional relationship between SOT and the microbiome and the ways in which SOT affects the microbiome and how these changes in turn shape the health of transplant patients. We also discuss proven 
and potential interventions for treating a disrupted microbiome and promising areas for future research.

\section{Introduction}

The past few decades have witnessed significant improvements in health outcomes following solid-organ transplantation (SOT). ${ }^{1-3}$ Early survival rates have risen substantially, even with the increasing use of organs from high-risk or marginal donors. This is largely attributed to medical and surgical advances, including increased efficacy of immunosuppressive therapies and reduced complications from posttransplant infections. ${ }^{4}$ However, in spite of these improvements, the long-term trajectory of survival post-SOT has failed to improve at a comparable rate. ${ }^{1-3,5-7}$ While numerous factors have been examined to explain this lack of clinical progress, a potential role for the human microbiome has recently been recognized. ${ }^{8-11}$ Particularly relevant to SOT is the relationship between the microbiome and the immune system, as the microbiome has been found to interact with and modulate the gut and systemic immune systems. ${ }^{9,12-14}$ End-stage organ disease followed by SOT can significantly perturb the human microbiome, with the potential to influence allograft and patient health adversely. This review explores the relationship between SOT and the microbiome, with a particular focus on bacteria, and discusses potential microbial-targeted interventions that could prevent detrimental alterations in the microbiome and improve outcomes for patients after SOT. We examine clinical, preclinical, and animal-based studies, since the results of animal research have the potential to inform future human studies. Animal studies allow for the specific investigation of various aspects of the SOT process in isolation (eg, the effect of individual antibiotics or immunosuppressants) in models with consistent genetic and microbial backgrounds and contain few of the confounding variables (eg, environment, diet, and medication regimes) that complicate human clinical research on the microbiome.

\section{The human microbiome}

Although the impact of commensal microorganisms on transplant health outcomes has been postulated since $1978,{ }^{15}$ the capability for studying these microorganisms on a large scale has emerged only over the last decade. The development of inexpensive and widely accessible technologies, including next-generation DNA sequencing, highly sensitive instruments for metabolomics, and powerful computing resources for bioinformatics, has led to a rapid expansion of the field of microbiome research ${ }^{16,17}$ (Figure 1). With it has come an explosion of discoveries documenting the significance of the

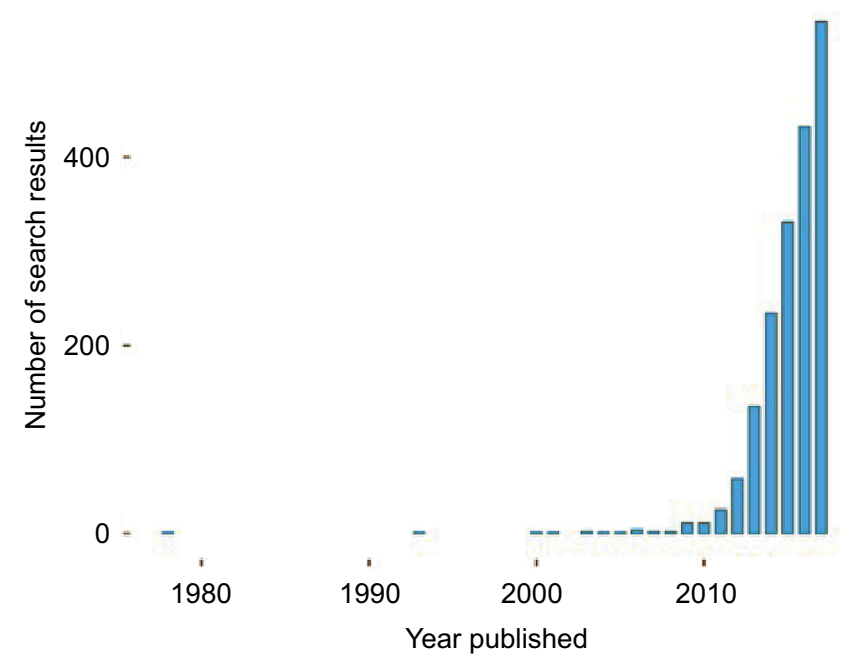

Figure I PubMed search results returned per year for articles relating to transplantation and the microbiome as of December 4, 2017.

Note: Search conducted using the keywords ((transplant) OR transplantation) AND microbiome.

microbiome in nearly all aspects of human health, including xenobiotic metabolism, biosynthesis of nutrients, and development and modification of the immune system. ${ }^{18,19}$

The human microbiome is defined as the collection of all microorganisms, including bacteria, archaea, fungi, and viruses, that exist in the nonsterile sites of the body, including the skin, respiratory tract, and gastrointestinal tract. ${ }^{20}$ The ratio of microbial to human cells is estimated to be 1.3-2.3:1, with the ratio of genetic content estimated to be an astounding 150:1. ${ }^{21,22}$ Microbial composition is highly variable between individuals and shaped by such factors as age, diet, medications, lifestyle, illness, and stress. ${ }^{23}$ However, functionality appears to be quite homogeneous among individuals, indicating that the conservation of function, and not necessarily composition, may be the most important parameter for assessing changes in the microbiome (Figure 2). ${ }^{24,25}$ Disruption of the homeostatic state of the microbiome has been implicated in a number of diseases, many of which affect patients after SOT. 19,26,27 Patient experiences, exposure, and treatment before and after SOT have the potential adversely to affect the microbiome and thereby influence their health outcomes.

\section{Impact of SOT on the microbiome}

Although functionality may turn out to be the most important parameter in assessing the microbiome, to date the composition of the microbiome has been studied most intensively. Shifts in microbial populations can occur in patients undergoing SOT, but high variability between transplant type and individual patients has been noted. In a study by Charlson et al, ${ }^{28}$ lung-transplant recipients were found to have higher 


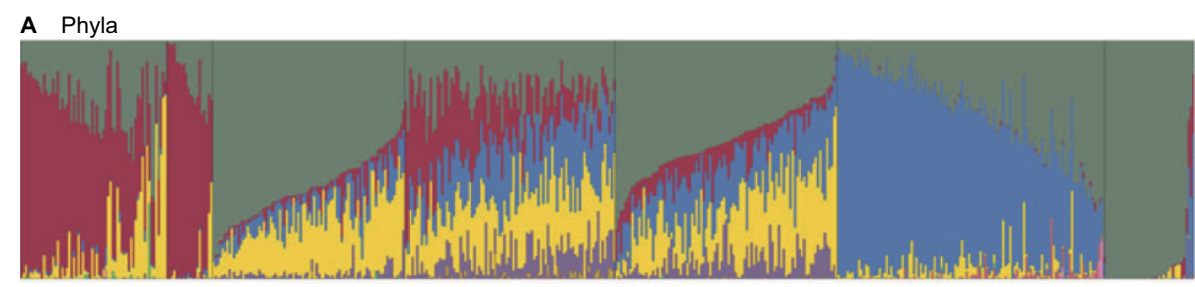

B Metabolic pathways

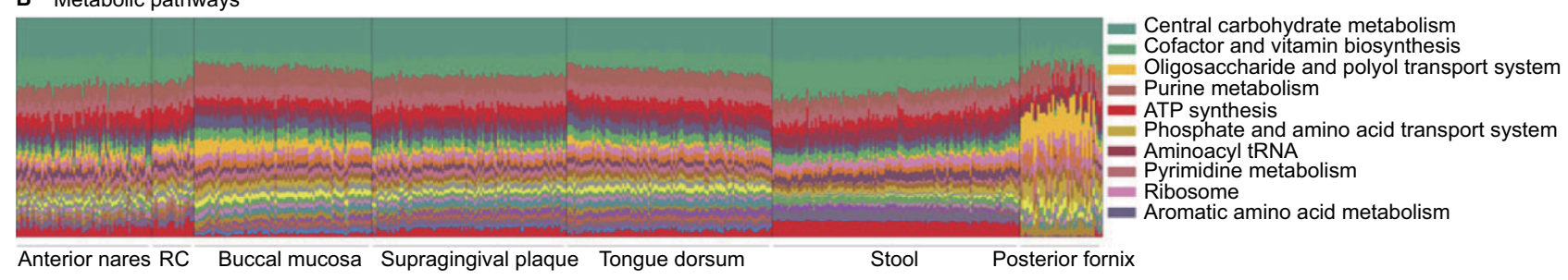

Figure 2 The human microbiome exhibits high body site (clusters along $x$-axis) and individual (individual columns) variation but functional diversity is highly conserved across both body site and individuals.

Notes: Height of bars denotes abundance of $(\mathbf{A})$ bacterial phyla and $(\mathbf{B})$ abundance of bacterial genes related to each metabolic pathway. Reprinted by permission from Springer Nature. Human Microbiome Project Consortium. Structure, function and diversity of the healthy human microbiome. Nature. 2012;486(7402):207-2 14. ${ }^{24}$

Abbreviation: RC, retroauricular crease.

bacterial and fungal loads in the lower respiratory tract compared to healthy controls. Also, their bacterial and fungal communities were shown to be less diverse and/or distinct, with outlier populations present more often in patients than in nontransplant controls. ${ }^{28}$ Liver-transplant patients have been found to have reduced fecal abundance of Bifidobacterium spp., Lactobacillus spp., and Faecalibacterium prausnitzii, but a higher abundance of Enterobacteriaceae and Enterococcus spp. compared to controls. ${ }^{29}$ In kidney-transplant patients, taxonomic shifts in commensal bacteria have been observed 1-month posttransplant with significant interindividual variation..$^{30}$ Due to the high degree of variation, as well as uncertainty about what precisely comprises a healthy microbiome,${ }^{24}$ longitudinal changes in the microbiota for a specific patient may be a more reliable predictor of health outcome than interindividual comparisons..$^{30}$ Indeed, compositional transformations are highly variable, depending on both the type of transplant and the patient themselves, emphasizing the multifactorial nature of this issue ${ }^{30-33}$ Known factors that have a role in post-SOT microbiome modifications include surgical procedures and the use of antibiotic prophylaxis and immunosuppressive drugs (ISDs).

\section{The effect of surgical procedures on the microbiome}

In addition to the effects of antibiotics, the transplantation procedure itself has been found to induce microbial alterations. Hartman et $\mathrm{al}^{34}$ demonstrated that patients who underwent an ileostomy experienced a shift in the ileal microbiome from strict anaerobes (eg, Bacteroidetes and Clostridia) to facultative anaerobes (eg, Lactobacilli and Enterobacteriaceae). This shift occurred because the ileostomy procedure allows oxygen into the small bowel, thereby encouraging the growth of organisms capable of producing ATP using aerobic respiration. This shift occurred in both transplant and nontransplant patients, suggesting it was an effect of the surgery itself, as opposed to other transplant-related factors. ${ }^{34}$ Donated organs are not sterilized prior to implantation, and thus possess their own foreign microbiome. Given the interindividual variability of the microbiome, the introduction of donor microbes could perturb the homeostasis of the recipient and possibly contribute to impaired graft function. Indeed, this complication has been demonstrated in lung-transplant recipients, where the development of primary graft dysfunction was correlated with the abundance of Acinetobacter in the lung microbiome of the donor. ${ }^{35}$

\section{The effect of antimicrobial prophylaxis on the microbiome}

Broad-spectrum antibiotic prophylaxis may be utilized posttransplantation to prevent perioperative complications arising due to the inherent increased risk of infection from the combination of surgery and systemic immunosuppression. However, this broad-spectrum approach does not target pathogenic organisms specifically and results in off-target depletion of many beneficial commensal bacteria. For example, Hill et $\mathrm{al}^{36}$ administered ampicillin, gentamicin, metronidazole, neomycin, and vancomycin, antibiotics commonly used in the perioperative period of $\mathrm{SOT}^{37}$ to mice via their drinking water, which resulted in a 10 -fold decrease in total bacterial 
abundance and large-scale community changes, including decreases in Firmicutes and increases in members of Bacteroidetes and Proteobacteria phyla. However, specific alterations varied with the type and duration of antibiotic treatment. It was noted that a reduction in mucosal-associated microbes in the intestine coincided with lower production of interferon gamma and interleukin (IL17A) by mucosal CD4-positive T cells, reducing the potential immunological response to infection. ${ }^{36}$

Individual antibiotics can also induce large-scale microbial changes. For example, vancomycin administered to mice in the drinking water decreased the abundance of Firmicutes and Bacteroidetes, ${ }^{38}$ oral ciprofloxacin reduced bacterial richness in the intestine by $30 \%,{ }^{36}$ and oral azithromycin exposure was found to diminish microbial richness and decrease the prevalence of Proteobacteria and Verrucomicrobia. ${ }^{39}$ Altering the gut microbiome with antibiotics not only reduces microbial diversity but also impairs the metabolic potential of the microbiome. A single high dose of oral streptomycin has been found to lower the quantity of fecal microbiota and decrease levels of bacterial metabolites by $87 \%$ through effects on the metabolism of carbohydrates, fatty acids, amino acids, steroids, and eicosanoids. ${ }^{40}$ Furthermore, an antibiotic-depleted gut microbiome can actually increase susceptibility to infection by decreasing the potential for colonization resistance by resident intestinal fauna. Colonization resistance refers to the ability of the normal gut microbiota to prevent the colonization and proliferation of microbes, usually pathogenic, that are not normally found in the gut or persist only at low densities. ${ }^{41}$ This is accomplished primarily through commensals outcompeting invading bacteria for nutrients, ${ }^{42}$ preventing proliferation by producing antimicrobial compounds ${ }^{43}$ and triggering the host immune system to regulate bacterial growth, ${ }^{44}$ ultimately maintaining homeostasis within the gut. Depleting the gut of resident microbes opens the door for pathogens to flourish, as is commonly observed in antibiotic-associated diarrhea. Antibiotic-associated diarrhea is caused by the proliferation of pathogenic bacteria, such as Clostridium difficile, a process facilitated by the antibioticmediated depletion of normal commensal bacteria. ${ }^{45}$ In immunosuppressed patients, this problem is compounded by a reduced ability to clear bacterial infections, which may lead to recurrent or refractory disease..$^{46-49}$

\section{The effect of ISDs on the microbiome}

The increased efficacy of modern ISDs to limit graft-rejection events after SOT is a major contributor to the increase in short-term survival post-SOT. ${ }^{4}$ However, these ISDs have been found to have a potentially negative influence on the microbiome. ${ }^{50-53}$ Tourret et $\mathrm{al}^{51}$ demonstrated that prednisolone consistently reduced the ratio of Bacteroidetes to Firmicutes phyla. While composition was also altered significantly at the family level, high variability among biological replicates was noted, indicating prednisolone acted aspecifically. Tacrolimus, everolimus, and prednisolone, but not mycophenolate mofetil, resulted in decreased expression of C-type lectin, an antimicrobial peptide, in the ileum. This suggests that the relationship between the host and the gut microbiota was disrupted, which possibly contributed to the observed overgrowth of toxic Escherichia coli. ${ }^{51}$ Compositional changes in the microbiome of nonhuman primates, specifically in the Enterobacteriales, Clostridiales, and Lactobacillales orders and Prevotella and Faecalibacterium genera, have been found to occur after treatment with alemtuzumab, a humanized monoclonal antibody used in SOT as an induction therapy to deplete lymphocytes. The authors attributed this effect to ablation of T-cell receptor $\alpha \beta^{+}$and $\gamma \delta^{+}$T cells, demonstrating an association between mucosal lymphocytes and bacterial species richness in the gut. ${ }^{54}$ In the salivary microbiome of renal and cardiac transplant patients undergoing chronic ( $\geq 1$ year) immunosuppression using various combinations of prednisone, mycophenolate mofetil, tacrolimus, sirolimus, cyclosporine, and azathioprine, an expansion of opportunistic pathogens, such as Pseudomonas aeruginosa, Acinetobacter baumannii, Staphylococcus aureus, and Enterococcus faecalis, was observed. ${ }^{50}$ This occurred despite a similar overall community structure between patients and controls, suggesting that microbial community function, drug effects, or specific bacterial subpopulations, and not simply overall composition, contributed to the permissive nature of the oral environment for pathogen colonization after SOT. ${ }^{50}$

\section{End-stage organ disease and the microbiome}

Microbiome disruption is not only caused by the transplant surgery and essential posttransplant medications but also likely begins even before transplantation as a result of the end-stage disease that necessitated the SOT. Vaziri et al ${ }^{55}$ found a significant difference in 190 bacterial operational taxonomic units between patients with end-stage renal disease and controls, with notable increases in the Actinobacteria, Proteobacteria, and Firmicutes phyla in patients with severe kidney disease. This effect was attributed mostly to the influx of urea, uric acid, and oxalate into the colon in these uremic patients, which altered the biochemical environment of the colon, as well as disrupting the colonic epithelial tight junc- 
tions. ${ }^{55,56}$ In patients with liver cirrhosis, there are significant decreases in the ratio of normal commensal to potentially pathogenic bacteria in the gut compared to controls. ${ }^{57}$ In a separate study, compositional differences characterized by decreased species richness and reduced abundance of Proteobacteria and Fusobacteria in cirrhotic patients were also seen. ${ }^{58}$ Functionally, cirrhotic patients' bacterial metagenomes were enriched for membrane transport orthologues, and an increase in pathway modules responsible for ammonia production was also seen, suggesting that bacteria play a role in the hyperammonemia complicating liver cirrhosis. ${ }^{58}$

\section{Impact of the microbiome on SOT patients}

It is important to note that the relationship between transplantation and the microbiome is not unidirectional but is instead bidirectional, complex, and constantly evolving. Therefore, altering a transplant patient's microbiome composition and function will potentially modify how the microbiome influences the host (Figure 3). The microbial disruptions occurring from SOT which have been described thus far, particularly in the gut, are predicted to exert a negative influence on the host. Changes in community structure and function can impair the ability of the gut microbiome to produce nutrients for the host, ${ }^{59}$ protect the host from colonization by pathogens, ${ }^{28}$ and induce protective inflammation. ${ }^{60,61}$ These factors can increase the risk of immune-related complications postSOT, an idea supported by the observed correlation between microbial disruption and rejection events. ${ }^{62-64}$

Bacterial dysbiosis was correlated with the rejection of small-bowel allografts in a study by $\mathrm{Oh}$ et al, ${ }^{62}$ which found that the abundance of ileal Firmicutes was lower in transplant patients experiencing rejection, whereas the abundance of Proteobacteria was higher. As well, the prevalence of

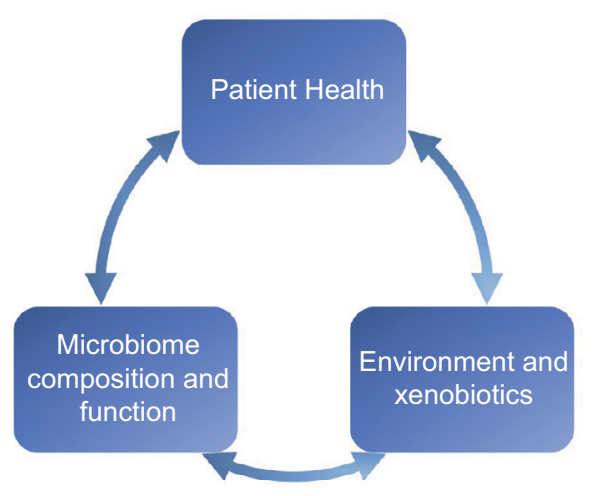

Figure 3 Bidirectional relationships between the environment and xenobiotics (including diet, lifestyle, and medications) and host microbiome and patient health. Note: Disruption of one variable likely to impact the others.
Escherichia and Klebsiella spp. was comparable in prerejection and active rejection samples but was distinct from samples obtained from patients with no evidence of rejection. This study reported no significant changes in the microbiome of small-bowel-transplant patients who were administered antibiotics compared with those who were not, and thus these microbiome alterations appeared to result from factors other than antibiotic treatment. ${ }^{62}$ In lung-transplant patients, alterations to pretransplant flora have been linked with the onset of bronchiolitis obliterans syndrome, one of the main causes of allograft loss after lung transplantation. ${ }^{65}$ Recolonization of the lung microbiome by resident pre-SOT bacteria, specifically the reestablishment of certain Pseudomonas spp., is protective for the development of bronchiolitis obliterans syndrome. ${ }^{63,66}$ Moreover, lung-transplant patients colonized with Simkania negevensis in their bronchoalveolar lavage microbiome have been found to be 3.4-fold more likely to develop allograft rejection. The presence of $S$. negevensis preceded allograft rejection in $37 \%$ of lung-transplant patients, which suggests a potential causal relationship between this bacterium and rejection events. ${ }^{64}$

While microbial dysbiosis influences rejection via many different mechanisms, the most significant may be the triggering of local and systemic inflammation. ${ }^{67,68}$ The observation that organs with a lower bacterial load, namely the heart and kidney, tend to have lower rejection rates than organs with higher exposure to commensal microorganisms and greater exposure to the external environment (skin, intestines, and lungs $)^{69}$ potentially supports this hypothesis. ${ }^{62-64,70-73}$

\section{Microbial-mediated immune-cell abnormalities posttransplantation}

The goal of immunosuppression after SOT is the downregulation of adaptive immunity to prevent allorejection, but this can be counteracted by the priming of recipient $T$ cells by gut microbes. In a mouse skin-graft model, both the bacterial pathogen Listeria monocytogenes and the commensal bacteria Staphylococcus aureus have the ability to stimulate and activate alloreactive $T$ cells in a specific-pathogen-free mouse skin-graft model via the activation of antigen-presenting cells and the production of IL6, respectively. ${ }^{73,74}$ Importantly, these mechanisms of activation occur systemically, not only locally to the colonized tissue, suggesting that the gut microbiome is able to affect alloimmunity of distant, sterile, transplanted organs. This view is supported by a study from Lei et al, ${ }^{75}$ where it was demonstrated that pretreating specific-pathogenfree mice with broad-spectrum antibiotics (gentamicin, kanamycin, colistin, metronidazole, and vancomycin) via 
oral gavage reduced the potential for T-cell priming, as indicated by delayed rejection events of both skin and cardiac allografts. ${ }^{75}$ The eukaryotic parasite Leishmania major was also found to expedite allograft rejection via priming of cross-reactive $T$ cells, despite the administration of anti-CD40 ligand antibody as costimulatory blockade, showing this phenomenon is not restricted to bacteria. ${ }^{76}$ Spleen cells collected from these mice were found to produce interferon gamma at a significantly higher rate than controls, indicating a high level of immunoactivity. ${ }^{76}$ Interestingly, the activation of the adaptive immune system by T-cell priming occurs mostly through Toll-like receptor (TLR) signaling and subsequent activation of the MyD88 pathway.

Evidence demonstrates that TLR signaling plays an important role in immune system activation after SOT. TLR signaling through the MyD88 pathway has been linked with increased allograft rejection. ${ }^{72,73,77}$ The lower incidence of allograft rejection in individuals with variants in TLR4 further suggests that activation of this receptor is involved in allorecognition. ${ }^{71}$ Alterations in the gut microbiome can also activate the TLR4-signaling pathway, which stimulates an innate inflammatory immune response and dysregulates the intestinal epithelial barrier, leading to increased inflammation. ${ }^{70}$ TLR4 signaling has also been found to dysregulate the gut microbiome through the altered expression of antimicrobial peptides, resulting in a feedback loop of gut microbial modulation. ${ }^{78}$

Regulatory $\mathrm{T}$ cells $\left(\mathrm{T}_{\text {regs }}\right)$ are immunosuppressive $\mathrm{T}$ cells that function to regulate the activity and proliferation of effector $\mathrm{T}$ cells and prevent autoimmune activity through the maintenance of tolerance to self-antigens. ${ }^{79}$ Researchers have established that antigens derived from certain microbial strains can impact the development of $\mathrm{T}_{\text {regs }}$ so as to promote tissue injury and alloreactive responses ${ }^{80}$ Microbial antigens can suppress retinoic acid, thereby hindering the development of induced $\mathrm{T}_{\text {regs }}\left(\mathrm{iT}_{\text {regs }}\right),{ }^{81}$ as retinoic acid is required by these cells for TGF $\beta$ signaling. ${ }^{82}$ Since $i \mathrm{~T}_{\text {regs }}$ can counteract tissue injury by inhibiting mucosal $\mathrm{T}_{\mathrm{H}} 2$ responses, ${ }^{83}$ the inability of $i \mathrm{~T}_{\text {regs }}$ to develop may contribute to allograft rejection. Brandl et $\mathrm{al}^{84}$ also demonstrated that dysbiosis induced by oral administration of vancomycin can modulate $T_{\text {reg }}$ phenotype to increase the likelihood of proinflammatory alloreactive T-cell responses, which demonstrates additional unintended complications of posttransplant antibiotics.

Immunoactivation by bacteria can also be organ specific. In liver transplantation, the release of microbe-associated molecular patterns, such as flagellin, into the portal venous circulation was found to shape immunoactivity by influencing the expression of major histocompatibility complex II expression (increased) and phagocytic activity (decreased) of Kupffer cells, which are liver-specific macrophages. This Kupffer cell phenotype has the potential to lead to increased risk of reperfusion injury and reduced bacterial clearance from the liver, compromising health outcomes after transplantation. ${ }^{85}$

\section{Additional consequences of dysbiosis on posttransplant outcomes}

Allorejection is not the only adverse event caused by microbial dysbiosis that may be hindering long-term survival post-SOT. Lu et $\mathrm{al}^{32}$ found that patients who experienced significant changes in intestinal flora following liver transplantation, including shifts toward more pathogenic bacteria and a loss in overall microbial diversity, had longer hospital stays and more severe infections. These microbial imbalances were largely attributed to prophylactic antibiotic administration during transplantation. ${ }^{32}$ Loss of microbial diversity following transplantation has also been correlated with posttransplant diarrhea related to decreased abundance of Bacteroidetes, Ruminococcus spp., Coprococcus spp., and Dorea spp. ${ }^{86}$

Drug metabolism research suggests that microbial changes post-SOT can also alter the impact of host metabolism on therapeutics, ${ }^{87}$ and these effects also extend to the ISDs, including tacrolimus. Patients with a greater quantity of fecal Faecalibacterium prausnitzii required higher doses of tacrolimus to obtain the same therapeutic effect as patients without dysbiosis. ${ }^{88}$ Optimizing a patient's immunosuppression is a great challenge, and the differential metabolism of ISDs due to varying microbial composition further complicates this important issue.

\section{Solutions and prevention}

Many precautions for protecting the microbiome are already standard practice in SOT, albeit mostly inadvertently. The majority of these safeguards are aimed at preventing pathological bacterial infections. However, as previously discussed, while antibiotics serve to remove harmful pathogens and may even help promote graft survival through the elimination of pathobionts, ${ }^{75}$ the often untargeted nature of antibiotic usage may deplete commensal bacteria and produce a less diverse microbial environment that is then primed for pathogen colonization. ${ }^{89}$ However, the outcome of antibiotic prophylaxis for an individual patient likely depends on the original or current composition of their microbiome and myriad other factors, not all of which are possible to control. 
A relatively new but increasingly considered treatment is synbiotic therapy, the concurrent administration of prebiotics and probiotics. ${ }^{90}$ Prebiotics are compounds with the potential to promote growth of healthy gut bacteria, whereas probiotics are live bacteria thought to be beneficial to health. ${ }^{91}$ While still quite broad in nature, synbiotic therapy aims to increase the diversity of the microbiome and restore proper function, as opposed to antibiotic therapy, which generally decreases diversity and impairs function. Synbiotic therapy following liver transplantation, specifically the administration of live Bifidobacterium breve and Lactobacillus casei bacteria along with indigestible galacto-oligosaccharides, reduced systemic inflammation 6-fold and decreased the rate of bacterial infections posttransplantation in a study of 50 patients randomized to receive either symbiotic therapy ( 2 days preoperatively and 2 weeks postoperatively) or not. ${ }^{92}$

Even more targeted approaches may also be beneficial, such as supplementation with microbial metabolic products that are downregulated in a damaged microbiome. Best known are the short-chain fatty acids (SCFAs) which are end products of bacterial fermentation in the gut, and by acting to reduce inflammation play an essential role in gut health..$^{93}$ In patients with severe liver cirrhosis, Bajaj et $\mathrm{al}^{57}$ identified decreases in the abundance of beneficial taxa, such as Lachnospiraceae, Ruminococcaceae, and Clostridiales, bacteria known to produce SCFAs and also compete with pathogenic bacteria for nutrients. ${ }^{57,94}$ Therefore, a reduction in these bacteria could lead to more frequent pathogenic infections. Restoring proper levels of SCFAs in the gut through replacement therapy could potentially compensate for the reduction in SCFA-producing bacteria and restore an appropriate functional and metabolic balance. A host of other bacterial metabolites also play an important role in maintaining homeostasis between the intestinal microbiome and the immune system. Bacterial indoles produced from the metabolism of tryptophan are responsible for stimulating IL22 production by innate lymphoid cells, ${ }^{95}$ which in turn stimulates the production of mitogenic factors and antimicrobial peptides, promoting a healthy intestinal epithelial layer and regulation of the adjacent bacterial communities. ${ }^{96,97}$ Bacteria-derived polysaccharide $\mathrm{A}$ has been found to stimulate the production of $\mathrm{T}_{\text {reg }}$ cells that release IL10, limiting IL17-induced inflammation. ${ }^{98}$ Also, the stimulation of NLRP6 by bacteria-derived metabolites taurine, histamine, and spermine maintains proper function of colon-derived inflammasomes, subsequent antimicrobial peptide production, and microbiome homeostasis. ${ }^{99}$ Supplementing a dysbiotic intestinal environment with such bacteria-derived metabolites as these may be a feasible method of controlling inflammation and aberrant bacterial growth, possibly helping to restore a structurally and functionally homeostatic microbiome.

Nonmicrobial interventions may also play a role in protecting the microbiome post-SOT. An inadvertent precaution taken against microbial dysbiosis is ischemic preconditioning, alternating periods of ischemia and reperfusion, performed to condition transplanted tissues to periods of restricted blood flow prior to organ procurement. ${ }^{100}$ Ischemic preconditioning prior to liver transplantation has been shown to restore pretransplant microbial composition, increase species diversity and richness, and decrease hepatic graft injury. ${ }^{101}$

As researchers gain further insight into the influence of the microbiome on post-SOT health outcomes, it will be increasingly possible to develop targeted approaches to prevent microbial dysbiosis. One such approach may be the use of fecal microbiota transplantation (FMT) to restore the microbiome after the perturbations experienced before and during SOT. FMT has already proven to be highly successful in treating recurrent $C$. difficile, an infection attributed to microbiome dysbiosis. ${ }^{102}$ Given that FMT has been increasingly considered as a treatment in other diseases involving microbial dysbiosis (eg, metabolic syndrome and inflammatory bowel disease), ${ }^{103}$ it may be fruitful to investigate the use of FMT to improve patient health post-SOT. Indeed, FMT has been utilized successfully in treating recurrent $C$. difficile infection in a pediatric patient (Figure 4).$^{49}$ Early recognition of the potential need for SOT could allow for the collection and storage of patients' own stool samples while their microbiome is still relatively stable, allowing for autologous FMT, ${ }^{104}$ or if their dysbiosis is advanced due to their end-stage disease (see previous section), then a healthy donor could be identified.

Although there are many potential interventions to restore a dysfunctional microbiome, preventive measures to avoid microbial dysbiosis in the first place would be ideal. Limiting the unnecessary use of broad-spectrum antibiotics is feasible today, but in the future, profiling a patient's microbiome prior to a planned intervention or initiation of a drug may be useful. Knowledge regarding the composition and function of a patient's microbiome could inform the transplant team about the potential risk for infection, rejection, unexpected medication metabolism, and other potential complications as a component of personalized transplant medicine. ${ }^{61}$

\section{Limitations of microbiome research}

Despite the explosion of knowledge regarding the human microbiome over the last decade, our knowledge in the field is far from complete, due in part to many technological limitations. These include issues with DNA-sequencing 


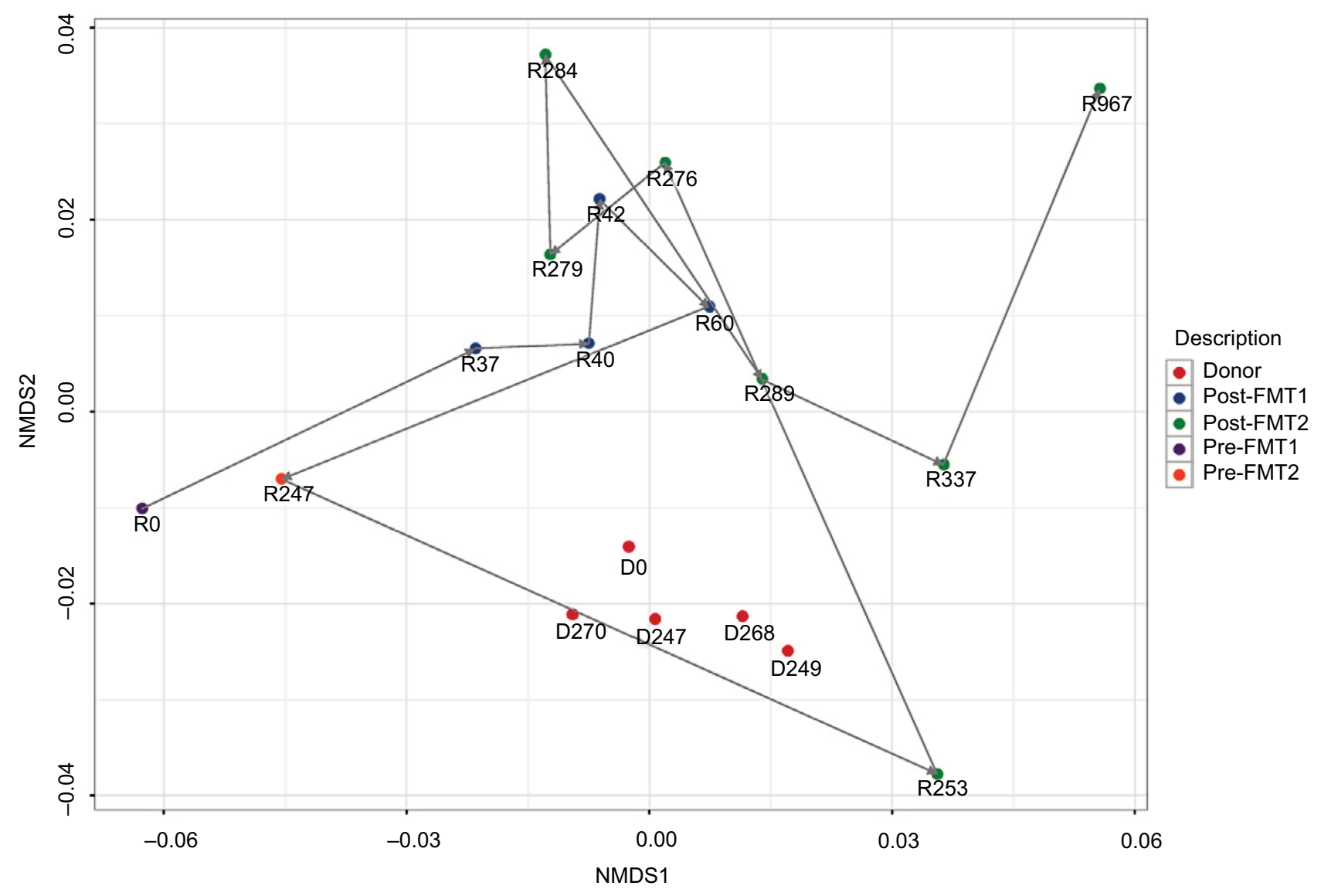

Figure 4 Changes in bacterial composition ( $\beta$-diversity) before and after two fecal microbiota transplants (FMTs) were used to treat refractory Clostridium difficile infections in a pediatric heart-transplant patient.

Notes: Clustered red circles indicate the stable donor microbiome. The purple circle represents the sample obtained before the first FMT, followed by blue circles (postFMTI). The patient relapsed (orange circle) after a course of antibiotics and was then treated with a second FMT. Following the second FMT, although the $C$. difficile infection did not recur, the microbiome demonstrated dynamic changes that may in part have been related to the use of mycophenolate mofetil. Reproduced from Flannigan KL, Rajbar $\mathrm{T}$, Moffat A, et al. Changes in composition of the gut bacterial microbiome after fecal microbiota transplantation for recurrent Clostridium difficile infection in a pediatric heart transplant patient. Front Cardiovasc Med. 2017;4:17. Creative Commons license and disclaimer available from: http://creativecommons.org/licenses/by/4.0/legalcode"http:// creativecommons.org/licenses/by/4.0/legalcode. ${ }^{49}$

techniques, ${ }^{105}$ a lack of understanding of the connections between microbial composition, genomic content, and metabolic function, ${ }^{24}$ as well as confounding factors associated with human disease and the use of animals to model human diseases. ${ }^{106}$ Moreover, a lack of consistency or agreement upon which techniques are best to use for microbiome studies produces high interstudy variability and confounds comparisons. ${ }^{107}$ Ultimately, more time is required to resolve these issues and for significant progress to be made in the field. However, the potential for treating the microbiome as a component of transplant medicine is significant and may be key to improving patient health and prolonging survival.

\section{Conclusion}

Collected knowledge from human and animal studies and relatively small trials suggest that the human microbiome may be a significant and previously overlooked environmental factor impacting the long-term health outcomes of transplant recipients. SOT has been shown to modulate both the compositional structure and the function of the human microbiome, and in turn, alterations in the microbiota can contribute to allograft rejection and the development of post-SOT comorbidities. While few proven interventions currently exist for treating a dysbiotic microbiome, research in the field is accelerating. At present, recognition that SOT has the potential to disrupt the important homeostasis that exists between the human host and their microbiome and efforts to minimize or correct these disruptions may be helpful in optimizing patient and allograft health.

\section{Acknowledgments}

Sources of funding included the Department of Pediatrics, Alberta Children's Hospital Research Institute, and the Libin Cardiovascular Institute of Alberta to SCG.

\section{Disclosure}

The authors report no conflicts of interest in this work. 


\section{References}

1. US Health Resources and Services Administration. Organ Procurement and Transplantation Network (OPTN) and Scientific Registry of Transplant Recipients (SRTR): OPTN/SRTR 2012 Annual Data Report. Rockville: HRSA; 2014.

2. Dipchand AI, Kirk R, Edwards LB, et al. Registry of the International Society for Heart and Lung Transplantation sixteenth official pediatric heart transplantation report-2013: focus theme-age. J Heart Lung Transplant. 2013;32(10):979-988.

3. Lund LH, Edwards LB, Kucheryavaya AY, et al. Registry of the International Society for Heart and Lung Transplantation thirtieth official adult heart transplant report—2013: focus theme-age. J Heart Lung Transplant. 2013;32(10):951-964.

4. Lodhi SA, Lamb KE, Meier-Kriesche HU. Solid organ allograft survival improvement in the United States: the long-term does not mirror the dramatic short-term success. Am J Transplant. 2011;11(6):1226-1235.

5. Pascual M, Theruvath T, Kawai T, Tolkoff-Rubin N, Cosimi AB. Strategies to improve long-term outcomes after renal transplantation. N Engl J Med. 2002;346(8):580-590.

6. Patel AK, Samaniego M. The struggle for optimization of long-term outcomes after kidney transplantation. Adv Chronic Kidney Dis. 2016;23(5):280.

7. Kim JJ, Marks SD. Long-term outcomes of children after solid organ transplantation. Clinics (Sao Paulo). 2014;69(Suppl 1):28-38.

8. Wang W, Xu S, Ren Z, Jiang J, Zheng S. Gut microbiota and allogeneic transplantation. J Transl Med. 2015;13:275.

9. Nellore A, Fishman JA. The microbiome, systemic immune function, and allotransplantation. Clin Microbiol Rev. 2015;29(1):191-199.

10. Vindigni SM, Surawicz CM. The gut microbiome: a clinically significant player in transplantation? Expert Rev Clin Immunol. 2015;11(7):781-783.

11. Alegre ML, Bartman C, Chong AS. Microbes and allogeneic transplantation. Transplantation. 2014;97(1):5-11.

12. Alegre ML, Mannon RB, Mannon PJ. The microbiota, the immune system and the allograft. Am J Transplant. 2014;14(6):1236-1248.

13. Nikoopour E, Singh B. Reciprocity in microbiome and immune system interactions and its implications in disease and health. Inflamm Allergy Drug Targets. 2014;13(2):94-104.

14. Clarke TB. Early innate immunity to bacterial infection in the lung is regulated systemically by the commensal microbiota via Nod-like receptor ligands. Infect Immun. 2014;82(11):4596-4606.

15. Brown JF, Balish E. Gastrointestinal microecology of BALB/c nude mice. Appl Environ Microbiol. 1978;36(1):144-159.

16. Mulcahy-O'Grady H, Workentine ML. The challenge and potential of metagenomics in the clinic. Front Immunol. 2016;7:29.

17. Knight R, Callewaert C, Marotz C, et al. The microbiome and human biology. Annu Rev Genomics Hum Genet. 2017;18:65-86.

18. Laukens D, Brinkman BM, Raes J, de Vos M, Vandenabeele P. Heterogeneity of the gut microbiome in mice: guidelines for optimizing experimental design. FEMS Microbiol Rev. 2016;36(40):117-132.

19. Cho I, Blaser MJ. The human microbiome: at the interface of health and disease. Nat Rev Genet. 2012;13(4):260-270.

20. Purchiaroni F, Tortora A, Gabrielli M, et al. The role of intestinal microbiota and the immune system. Eur Rev Med Pharmocol Sci. 2013;(17):323-333.

21. Sender R, Fuchs S, Milo R. Revised estimates for the number of human and bacteria cells in the body. PLoS Biol. 2016;14(8):e1002533.

22. Qin J, Li R, Raes J, et al. A human gut microbial gene catalog established by metagenomic sequencing. Nature. 2010;464(7285):59-65.

23. Benson AK, Kelly SA, Legge R, et al. Individuality in gut microbiota composition is a complex polygenic trait shaped by multiple environmental and host genetic factors. Proc NatlAcad Sci USA. 2010;107(44): 18933-18938.

24. Human Microbiome Project Consortium. Structure, function and diversity of the healthy human microbiome. Nature 2012;486(7402):207-214.
25. Ursell LK, Haiser HJ, van Treuren W, et al. The intestinal metabolome: an intersection between microbiota and host. Gastroenterology. 2014;146(6):1470-1476.

26. Hill DA, Siracusa MC, Abt MC, et al. Commensal bacteria-derived signals regulate basophil hematopoiesis and allergic inflammation. Nat Med. 2012;18(4):538-546.

27. Bhat M, Pasini E, Copeland J, et al. Impact of immunosuppression on the metagenomic composition of the intestinal microbiome: a systems biology approach to post-transplant diabetes. Sci Rep. 2017;7(1):10277.

28. Charlson ES, Diamond JM, Bittinger K, et al. Lung-enriched organisms and aberrant bacterial and fungal respiratory microbiota after lung transplant. Am J Respir Crit Care Med. 2012;186(6):536-545.

29. Wu ZW, Ling ZX, Lu HF, et al. Changes of gut bacteria and immune parameters in liver transplant recipients. Hepatobiliary Pancreat Dis Int. 2012;11(1):40-50

30. Fricke WF, Maddox C, Song Y, Bromberg JS. Human microbiota characterization in the course of renal transplantation. Am JTransplant. 2014;14(2):416-427.

31. Wang W, Xu S, Ren Z, Jiang J, Zheng S. Gut microbiota and allogeneic transplantation. J Transl Med. 2015;13:275.

32. $\mathrm{Lu} \mathrm{H}, \mathrm{He} \mathrm{J}, \mathrm{Wu} \mathrm{Z}$, et al. Assessment of microbiome variation during the perioperative period in liver transplant patients: a retrospective analysis. Microb Ecol. 2013;65(3):781-791.

33. Borewicz K, Pragman AA, Kim HB, Hertz M, Wendt C, Isaacson RE. Longitudinal analysis of the lung microbiome in lung transplantation. FEMS Microbiol Lett. 2013;339(1):57-65.

34. Hartman AL, Lough DM, Barupal DK, et al. Human gut microbiome adopts an alternative state following small bowel transplantation. Proc Natl Acad Sci U S A. 2009;106(40):17187-17192.

35. Xu Z, Schuessler R, Steward N, et al. Donor microbiota and its contribution for the development of primary graft dysfunction following human lung transplantation. Am J Transpl. 2015;15(Suppl 3).

36. Hill DA, Hoffmann C, Abt MC, et al. Metagenomic analyses reveal antibiotic-induced temporal and spatial changes in intestinal microbiota with associated alterations in immune cell homeostasis. Mucosal Immunol. 2010;3(2):148-158.

37. Anesi JA, Blumberg EA, Abbo LM. Perioperative antibiotic prophylaxis to prevent surgical site infections in solid organ transplantation. Transplantation. 2018;102(1):21-34.

38. Hwang I, Park YJ, Kim Y-R, et al. Alteration of gut microbiota by vancomycin and bacitracin improves insulin resistance via glucagon-like peptide 1 in diet-induced obesity. FASEB J. 2015;29(6): 2397-2411.

39. Parker EPK, Praharaj I, John J, et al. Changes in the intestinal microbiota following the administration of azithromycin in a randomised placebo-controlled trial among infants in south India. Sci Rep. 2017;7(1):9168.

40. Antunes LC, Han J, Ferreira RB, Lolić P, Borchers CH, Finlay BB. Effect of antibiotic treatment on the intestinal metabolome. Antimicrob Agents Chemother. 2011;55(4):1494-1503.

41. Lawley TD, Walker AW. Intestinal colonization resistance. Immunology. 2012;138(1):1-11

42. Momose Y, Hirayama K, Itoh K. Competition for proline between indigenous Escherichia coli and E. coli O157:H7 in gnotobiotic mice associated with infant intestinal microbiota and its contribution to the colonization resistance against $E$. coli $\mathrm{O} 157: \mathrm{H} 7$. Antonie Van Leeuwenhoek. 2008;94(2):165-171.

43. Rea MC, Sit CS, Clayton E, et al. Thuricin CD, a posttranslationally modified bacteriocin with a narrow spectrum of activity against Clostridium difficile. Proc Natl Acad Sci U SA. 2010;107(20):9352-9357.

44. Vaishnava S, Behrendt CL, Ismail AS, Eckmann L, Hooper L. Paneth cells directly sense gut commensals and maintain homeostasis at the intestinal host-microbial interface. Proc Natl Acad Sci U S A. 2008;105(52):20858-20863.

45. Varughese CA, Vakil NH, Phillips KM. Antibiotic-associated diarrhea J Pharm Pract. 2013;26(5):476-482. 
46. Honda H, Dubberke ER. Clostridium difficile infection in solid organ transplant recipients. Curr Opin Infect Dis. 2014;27(4):336-341.

47. Kelly CR, Ihunnah C, Fischer M, et al. Fecal microbiota transplant for treatment of Clostridium difficile infection in immunocompromised patients. Am J Gastroenterol. 2014;109(7):1065-1071.

48. Alonso CD, Kamboj M. Clostridium difficile infection (CDI) in solid organ and hematopoietic stem cell transplant recipients. Curr Infect Dis Rep. 2014;16(8):414.

49. Flannigan KL, Rajbar T, Moffat A, et al. Changes in composition of the gut bacterial microbiome after fecal microbiota transplantation for recurrent Clostridium difficile infection in a pediatric heart transplant patient. Front Cardiovasc Med. 2017;4:17.

50. Diaz PI, Hong BY, Frias-Lopez J, et al. Transplantation-associated long-term immunosuppression promotes oral colonization by potentially opportunistic pathogens without impacting other members of the salivary bacteriome. Clin Vaccine Immunol. 2013;20(6):920-930.

51. Tourret J, Willing BP, Dion S, MacPherson J, Denamur E, Finlay BB. Immunosuppressive treatment alters secretion of ileal antimicrobial peptides and gut microbiota, and favors subsequent colonization by uropathogenic Escherichia coli. Transplantation. 2017;101(1):74-82.

52. Ritter ML, Pirofski L. Mycophenolate mofetil: effects on cellular immune subsets, infectious complications, and antimicrobial activity. Transpl Infect Dis. 2009;11(4):290-297.

53. Kim HB, Wang Y, Sun X. A detrimental role of immunosuppressive drug, dexamethasone, during Clostridium difficile infection in association with a gastrointestinal microbial shift. J Microbiol Biotechnol. 2016;26(3):567-571.

54. Li QR, Wang CY, Tang C, He Q, Li N, Li JS. Reciprocal interaction between intestinal microbiota and mucosal lymphocyte in cynomolgus monkeys after alemtuzumab treatment. Am J Transplant. 2013;13(4):899-910.

55. Vaziri ND, Wong J, Pahl M, et al. Chronic kidney disease alters intestinal microbial flora. Kidney Int. 2013;83(2):308-315.

56. Vaziri ND, Yuan J, Rahimi A, Ni Z, Said H, Subramanian VS. Disintegration of colonic epithelial tight junction in uremia: a likely cause of CKD-associated inflammation. Nephrol Dial Transplant. 2012;27(7):2686-2693.

57. Bajaj JS, Heuman DM, Hylemon PB, et al. Altered profile of human gut microbiome is associated with cirrhosis and its complications. J Hepatol. 2014;60(5):940-947.

58. Qin N, Yang F, Li A, et al. Alterations of the human gut microbiome in liver cirrhosis. Nature. 2014;513(7516):59-64.

59. Tremaroli V, Bäckhed F. Functional interactions between the gut microbiota and host metabolism. Nature. 2012;489(7415):242-249.

60. Biedermann L, Rogler G. The intestinal microbiota: its role in health and disease. Eur J Pediatr. 2015;174(2):151-167.

61. Kashyap PC, Chia N, Nelson H, Segal E, Elinav E. Microbiome at the frontier of personalized medicine. Mayo Clin Proc. 2017;92(12): $1855-1864$.

62. Oh PL, Martínez I, Sun Y, Walter J, Peterson DA, Mercer DF. Characterization of the ileal microbiota in rejecting and nonrejecting recipients of small bowel transplants. Am J Transplant. 2012;12(3):753-762.

63. Willner DL, Hugenholtz P, Yerkovich ST, et al. Reestablishment of recipient-associated microbiota in the lung allograft is linked to reduced risk of bronchiolitis obliterans syndrome. Am J Respir Crit Care Med. 2013;187(6):640-647.

64. Husain S, Kahane S, Friedman MG, et al. Simkania negevensis in bronchoalveolar lavage of lung transplant recipients: a possible association with acute rejection. Transplantation. 2007;83(2):138-143.

65. Gottlieb J, Mattner F, Weissbrodt H, et al. Impact of graft colonization with Gram-negative bacteria after lung transplantation on the development of bronchiolitis obliterans syndrome in recipients with cystic fibrosis. Respir Med. 2009;103(5):743-749.

66. Dickson RP, Erb-Downward JR, Freeman CM, et al. Changes in the lung microbiome following lung transplantation include the emergence of two distinct $P$ seudomonas species with distinct clinical associations. PLoS One. 2014;9(5):e97214.
67. Ardalan M, Vahed SZ. Gut microbiota and renal transplant outcome. Biomed Pharmacother. 2017;90:229-236.

68. Chong AS, Alegre ML. The impact of infection and tissue damage in solid-organ transplantation. Nat Rev Immunol. 2012;12(6):459-471.

69. Lechler RI, Sykes M, Thomson AW, Turka LA. Organ transplantation: how much of the promise has been realized? Nat Med. 2005;11(6):605-613.

70. Caesar R, Tremaroli V, Kovatcheva-Datchary P, Cani PD, Bäckhed F. Crosstalk between gut microbiota and dietary lipids aggravates WAT inflammation through TLR signaling. Cell Metab. 2015;22(4):658-668.

71. Palmer SM, Burch LH, Davis RD, et al. The role of innate immunity in acute allograft rejection after lung transplantation. Am J Respir Crit Care Med. 2003;168(6):628-632.

72. Goldstein DR, Tesar BM, Akira S, Lakkis FG. Critical role of the Toll-like receptor signal adaptor protein MyD88 in acute allograft rejection. J Clin Invest. 2003;111(10):1571-1578.

73. Wang T, Chen L, Ahmed E, et al. Prevention of allograft tolerance by bacterial infection with Listeria monocytogenes. J Immunol. 2008;180(9):5991-5999.

74. Ahmed EB, Wang T, Daniels M, Alegre ML, Chong AS. IL-6 induced by Staphylococcus aureus infection prevents the induction of skin allograft acceptance in mice. Am J Transplant. 2011;11(5):936-946.

75. Lei YM, Chen L, Wang Y, et al. The composition of the microbiota modulates allograft rejection. J Clin Invest. 2016;126(7):2736-2744.

76. Pantenburg B, Heinzel F, Das L, Heeger PS, Valujskikh A. T cells primed by Leishmania major infection cross-react with alloantigens and alter the course of allograft rejection. J Immunol. 2002;169(7): 3686-3693.

77. Chen L, Wang T, Zhou P, et al. TLR engagement prevents transplantation tolerance. Am J Transplant. 2006;6(10):2282-2291.

78. Dheer R, Santaolalla R, Davies JM, et al. Intestinal epithelial Toll-like receptor 4 signaling affects epithelial function and colonic microbiota and promotes a risk for transmissible colitis. Am Soc Microbiol. 2016;84(3):798-810.

79. Sakaguchi $\mathrm{S}$. Naturally arising CD4+ regulatory $\mathrm{T}$ cells for immunologic self-tolerance and negative control of immune responses. Annu Rev Immunol. 2004;22:531-562.

80. Zeng H, Chi H. Metabolic control of regulatory T cell development and function. Trends Immunol. 2015;36(1):3-12.

81. Peck A, Mellins ED. Plasticity of T-cell phenotype and function: the T helper type 17 example. Immunology. 2010;129(2):147-153.

82. Mucida D, Park Y, Kim G, et al. Reciprocal Th17 and regulatory T cell differentiation mediated by retinoic acid. Science. 2007;317(5835): 256-260.

83. Geuking MB, Cahenzli J, Lawson MA, et al. Intestinal bacterial colonization induces mutualistic regulatory $\mathrm{T}$ cell responses. Immunity. 2011;34(5):794-806.

84. Brandl K, Plitas G, Mihu CN, et al. Vancomycin-resistant enterococci exploit antibiotic-induced innate immune deficits. Nature. 2008;455(7214):804-807.

85. Corbitt N, Kimura S, Isse K, et al. Gut bacteria drive Kupffer cell expansion via MAMP-mediated ICAM-1 induction on sinusoidal endothelium and influence preservation-reperfusion injury after orthotopic liver transplantation. Am J Pathol. 2013;182(1):180-191.

86. Lee JR, Muthukumar T, Dadhania D, et al. Gut microbial community structure and complications following kidney transplantation: a pilot study. Transplantation. 2014;98(7):697-705.

87. Vázquez-Baeza Y, Callewaert C, Debelius J, et al. Impacts of the human gut microbiome on therapeutics. Annu Rev Pharmacol Toxicol. 2018;58:253-270.

88. Lee JR, Muthukumar T, Dadhania D, et al. Gut microbiota and tacrolimus dosing in kidney transplantation. PLoS One. 2015;10(3):e0122399.

89. Chang JY, Antonopoulos DA, Kalra A, et al. Decreased diversity of the fecal microbiome in recurrent Clostridium difficile-associated diarrhea. J Infect Dis. 2008;197(3):435-438.

90. Patel R, DuPont HL. New approaches for bacteriotherapy: prebiotics, new-generation probiotics, and synbiotics. Clin Infect Dis. 2015;60 (Suppl 2):S108-S121. 
91. Petschow B, Doré J, Hibberd P, et al. Probiotics, prebiotics, and the host microbiome: the science of translation. Ann N Y Acad Sci. 2013;1306:1-17.

92. Eguchi S, Takatsuki M, Hidaka M, Soyama A, Ichikawa T, Kanematsu T. Perioperative synbiotic treatment to prevent infectious complications in patients after elective living donor liver transplantation: a prospective randomized study. Am J Surg. 2011;201(4):498-502.

93. Tan J, McKenzie C, Potamitis M, Thorburn AN, Mackay CR, Macia L. The role of short-chain fatty acids in health and disease. Adv Immunol. 2014;121:91-119.

94. Nava GM, Stappenbeck TS. Diversity of the autochthonous colonic microbiota. Gut Microbes. 2010;2(2):99-104.

95. Zelante $\mathrm{T}$, Iannitti RG, Cunha $\mathrm{C}$, et al. Tryptophan catabolites from microbiota engage aryl hydrocarbon receptor and balance mucosal reactivity via interleukin-22. Immunity. 2013;39(2):372-385.

96. Sonnenberg GF, Fouser LA, Artis D. Border patrol: regulation of immunity, inflammation and tissue homeostasis at barrier surfaces by IL-22. Nat Immunol. 2011;12(5):383-390.

97. Zheng Y, Valdez PA, Danilenko DM, et al. Interleukin-22 mediates early host defense against attaching and effacing bacterial pathogens. Nat Med. 2008;14(3):282-289.

98. Mazmanian SK, Round JL, Kasper DL. A microbial symbiosis factor prevents intestinal inflammatory disease. Nature. 2008;453(7195): $620-625$.

99. Levy M, Thaiss CA, Zeevi D, et al. Microbiota-modulated metabolites shape the intestinal microenvironment by regulating NLRP6 inflammasome signaling. Cell. 2015;163(6):1428-1443.
100. Raeburn CD, Zimmerman MA, Arya J, Barsness K, Harken AH. Ischemic preconditioning: fact or fantasy? J Card Surg. 2002;17(6): 536-542.

101. Ren Z, Cui G, Lu H, et al. Liver ischemic preconditioning (IPC) improves intestinal microbiota following liver transplantation in rats through $16 \mathrm{~s}$ rDNA-based analysis of microbial structure shift. PLoS One. 2013;8(10):e75950.

102. Lahtinen P, Mattila E, Anttila VJ, et al. Faecal microbiota transplantation in patients with Clostridium difficile and significant comorbidities as well as in patients with new indications: a case series. World $J$ Gastroenterol. 2017;23(39):7174-7184.

103. Gupta S, Allen-Vercoe E, Petrof EO. Fecal microbiota transplantation: in perspective. Therap Adv Gastroenterol. 2016;9(2):229-239.

104. Gundling F, Tiller M, Agha A, Schepp W, Iesalnieks I. Successful autologous fecal transplantation for chronic diversion colitis. Tech Coloproctol. 2015;19(1):51-52.

105. Poretsky R, Rodriguez LM, Luo C, Tsementzi D, Konstantinidis KT. Strengths and limitations of $16 \mathrm{~S}$ rRNA gene amplicon sequencing in revealing temporal microbial community dynamics. PLoS One. 2014;9(4):e93827.

106. Quigley EM. Gut microbiome as a clinical tool in gastrointestinal disease management: are we there yet? Nat Rev Gastroenterol Hepatol. 2017;14(5):315-320.

107. Hiergeist A, Reischl U, Gessner A. Multicenter quality assessment of $16 \mathrm{~S}$ ribosomal DNA-sequencing for microbiome analyses reveals high inter-center variability. Int J Med Microbiol. 2016;306(5):334-342.
Transplant Research and Risk Management

\section{Publish your work in this journal}

Transplant Research and Risk Management is an international, peerreviewed open access journal focusing on all aspects of transplantation and risk management to achieve optimal outcomes in the recipient improving survival and quality of life. The manuscript management system is completely online and includes a very quick and fair peer-review system,

\section{Dovepress}

which is all easy to use. Visit http://www.dovepress.com/testimonials.php to read real quotes from published authors. 